\title{
Inception of a national multidisciplinary registry for stereotactic radiosurgery
}

\author{
Jason P. Sheehan, MD, PhD, ${ }^{1}$ Brian D. Kavanagh, MD, MPH, ${ }^{2}$ Anthony Asher, MD, ${ }^{3}$ and \\ Robert E. Harbaugh, MD4
}

\begin{abstract}
1Department of Neurological Surgery, University of Virginia Health System, Charlottesville, Virginia; ${ }^{2}$ Department of Radiation Oncology, University of Colorado at Denver, Aurora, Colorado; ${ }^{3}$ Carolina Neurosurgery \& Spine, Charlotte, North Carolina; and ${ }^{4}$ Penn State Medical Center, Hershey, Pennsylvania
\end{abstract}

\begin{abstract}
Stereotactic radiosurgery (SRS) represents a multidisciplinary approach to the delivery of ionizing high-dose radiation to treat a wide variety of disorders. Much of the radiosurgical literature is based upon retrospective single-center studies along with a few randomized controlled clinical trials. More timely and effective evidence is needed to enhance the consistency and quality of and clinical outcomes achieved with SRS. The authors summarize the creation and implementation of a national SRS registry.

The American Association of Neurological Surgeons (AANS) through NeuroPoint Alliance, Inc., started a successful registry effort with its lumbar spine initiative. Following a similar approach, the AANS and NeuroPoint Alliance collaborated with corporate partners and the American Society for Radiation Oncology to devise a data dictionary for an SRS registry. Through administrative and financial support from professional societies and corporate partners, a framework for implementation of the registry was created. Initial plans were devised for a 3-year effort encompassing 30 high-volume SRS centers across the country. Device-specific web-based data-extraction platforms were built by the corporate partners. Data uploaders were then used to port the data to a common repository managed by Quintiles, a national and international health care trials company.

Audits of the data for completeness and veracity will be undertaken by Quintiles to ensure data fidelity. Data governance and analysis are overseen by an SRS board comprising equal numbers of representatives from the AANS and NeuroPoint Alliance. Over time, quality outcome assessments and post hoc research can be performed to advance the field of SRS.

Stereotactic radiosurgery offers a high-technology approach to treating complex intracranial disorders. Improvements in the consistency and quality of care delivered to patients who undergo SRS should be afforded by the national registry effort that is underway.

http://thejns.org/doi/abs/10.3171/2015.1.JNS142466
\end{abstract}

KEY WORDS stereotactic radiosurgery; quality; registry

$\mathrm{S}$ INCE its conception in 1951, the field of stereotactic radiosurgery (SRS) has demonstrated substantial growth, and it has altered treatment paradigms for patients with complex and diverse intracranial disorders. ${ }^{12}$ In the United States alone, there are more than 100 distinct SRS centers that treat thousands of patients each year. Patients are treated for diverse indications such as benign and malignant brain tumors, vascular malformations, and functional disorders.
SRS is routinely delivered using several common radiosurgical devices. Despite the exponential growth of radiosurgery in the United States during the past 2 decades, the majority of the radiosurgical literature is composed of single-center retrospective studies.

There are, of course, a small percentage of retrospective multicenter pooled analyses and even fewer prospective randomized clinical trials (RCTs). Some prospective multicenter SRS trials, such as ACOSOG-N0574 and the

ABBREVIATIONS AANS = American Association of Neurological Surgeons; ABMS = American Board of Medical Specialties; ASTRO = American Society for Radiation Oncology; $\mathrm{MOC}=$ maintenance of certification; NPA = NeuroPoint Alliance; RCT = randomized clinical trial; SRS = stereotactic radiosurgery.

SUBMITTED October 30, 2014. ACCEPTED January 26, 2015.

INCLUDE WHEN CITING Published online August 7, 2015; DOI: 10.3171/2015.1.JNS142466. 
ROSE (Radiosurgery or Open Surgery for Epilepsy) trial, struggled for years to accrue adequate numbers of patients. Reasons for slow patient accrual include limited numbers of centers, low incidence of the underlying disease, patient unwillingness to be randomly assigned to undergo brain surgery, and clinical equipoise.

Although RCTs can often provide substantial high-level scientific information to a field, there are numerous logistical obstacles to their successful completion. The price for such trials can be on the order of millions of dollars for both direct and indirect costs, which makes them impractical to afford answers to the many questions that remain in the field of SRS. In a recent study, the average price per patient in an RCT was $\$ 47,000$, and some studies have costs as high as $\$ 85,000$ per patient. ${ }^{17}$ Bringing a new drug or device to the clinic requires $\$ 800$ million to $\$ 2$ billion, and RCTs are known to be a major driver in this cost. ${ }^{17}$

Prospective, observational registries have become important initiatives for quality improvement and patient safety, and there is a newfound appreciation for the "medicine-based evidence" that can be derived from these studies. ${ }^{7}$ A large prospectively collected patient database can also serve as a powerful tool for clinical research. Here, we summarize the development and implementation of a national SRS registry compatible with all contemporary delivery devices.

\section{Inception of the SRS Registry}

The American Association of Neurological Surgeons (AANS), under the leadership of Drs. Robert Harbaugh and Jon Robertson, launched the NeuroPoint Alliance (NPA) in 2008. The goal of the NPA is to collect, analyze, and report on the clinical data from US-based neurosurgical practices to ensure and enhance quality of care. Data collection through a prospective audited registry can also serve important research needs in a fashion that RCTs cannot. The NPA helped to bring together national stakeholders, including physicians, health care plans, health systems, and government agencies. ${ }^{2}$ In the first 5 months after launching the lumbar spine registry, 1357 patients from 21 sites were enrolled into the lumbar spine module. ${ }^{14}$ Of those patients who lived to the 3 -month postoperative time point, $90 \%$ had follow-up data enrolled in the registry. ${ }^{14}$ At approximately that same time point and in much the same fashion, the American Society for Radiation Oncology (ASTRO) and its Radiation Oncology Institute began to develop a prostate cancer registry. ${ }^{9}$

After the NPA demonstrated proof of principle with a highly successful lumbar spine registry and ASTRO embarked upon the National Radiation Oncology Registry, other indications for registries began to be explored. Stereotactic radiosurgery was a reasonable topic for a national registry. It has a fairly well-defined set of indications and a robust literature spanning more than 3 decades, thereby making the completion of new RCTs difficult. The SRS approach is a high-technology one, and it had seen fairly rapid growth in the past decade, which made its patient care volume appreciable enough for a registry. Significant variability in treatment indications, techniques, and technology exists within the field of SRS; a registry may help to reduce such variability. Also, there is a relatively nar- row field of key stakeholders for SRS, including neurosurgeons, radiation oncologists, health care payers, governmental agencies, and 3 major SRS-device manufacturers.

\section{Interdisciplinary Collaboration}

Stereotactic radiosurgery is performed in a multidisciplinary fashion. Leksell worked for many years with Larsson, a medical physicist, to build the original Gamma Knife unit. ${ }^{8}$ Around that same time, innovative work in the field of SRS using heavy particles from cyclotrons was conducted by Raymond Kjellberg and Jacob Fabrikant. In 1983 at a center in Buenos Aires, Argentina, Betti and Derechinsky ${ }^{4,5}$ developed the concept of a dedicated linear accelerator for SRS. That system relied upon a high-voltage linear accelerator, and it used a specialized chair and a Talairach stereotactic frame for the patient. ${ }^{10}$ A number of additional innovative developments in linear accelerator-based SRS devices followed from teams including Hartman and Sturm ${ }^{11}$ in Heidelberg, Germany, Winston and Lutz ${ }^{13}$ in Boston, Massachusetts, Colombo et al. ${ }^{6}$ in Vincenza, Italy, Barcia-Salorio in Valencia, Spain, and Podgorsak ${ }^{15}$ in Canada.

The definition sanctioned by the AANS, Congress of Neurological Surgeons, and ASTRO recommends radiosurgical delivery by a team consisting of a neurosurgeon, a radiation oncologist, and a medical physicist. ${ }^{3}$ Similarly, the American College of Radiology recommended a similar multidisciplinary approach to ensure quality of care and went so far as to detail specific responsibilities for the individual members of the multidisciplinary team during the SRS process. ${ }^{16}$

Because radiosurgery is performed by clinician partners (neurosurgeons and radiation oncologists), it was natural to extend this partnership to the registry. The AANS reached out to the ASTRO on the SRS registry project. Over time, a partnership for the national SRS registry was forged between the AANS and ASTRO. Both organizations created a joint governance board for the registry. The board comprised equal representation of neurosurgeons and radiation oncologists. Specific aims of the registry were established, including the following: 1) to define national patterns of care in radiosurgery and assess for gaps in treatment quality; 2) to provide benchmark data and quality-improvement tools for clinicians performing radiosurgery; 3 ) to yield information for clinicians and patients during the treatment process to support informed decision making; 4) to improve health care outcomes and potentially lower the cost of care delivered to patients who undergo radiosurgery; and 5) to provide longitudinal data for outcome-related SRS studies. In particular, the last aim will enable post hoc research queries to be performed with the prospectively collected quality data.

Then, the SRS board created a data dictionary, which is a central repository relating the data, origin, usage, and format of elements comprising the registry. The data dictionary includes nearly 135 separate fields, including patient attributes, radiosurgical parameters, preprocedural and postprocedural clinical features, and radiographic results. A sample of the more than 20-page data-dictionary document is provided in Table 1. Critical to the assessment 
of quality and outcomes was neuroimaging analysis. Target tumor volume response can now be measured through the use of imaging analytics applied to standardized Digital Imaging and Communications in Medicine (DICOM) data sets. Uniformity of outcome metrics and analytical tools enables more consistent data and greater validity for the aggregation of data across various SRS platforms and diverse SRS centers. For instance, one SRS team may prescribe a dose to a tumor with no margin, and another may prescribe a dose to a volume encompassing the tumor and a 1-mm, uniform margin. Data collected in the registry include dose-volume histogram information for the dose to a particular volume of the tumor and not the planned treatment volume with its 1-mm gross tumor volumeto-planned treatment volume expansion. Because gross tumor volume-to-planned treatment volume expansion may vary from case to case within an individual center or between different centers, dose-volume histogram data exported to the registry need to be normalized to the target volume and not the planned treatment volume. Consistency of registry data allows for a comparison of "apples to apples" rather than "apples to oranges."

Many elements in the data dictionary can be inserted into the registry database in a semiautomated or automated fashion. Semiautomated or automated population of fields in the registry can occur through data extraction from commonly used SRS software packages (e.g., iPlan or Gamma Plan). However, the registry is not device specific; the data elements are compatible with those in all currently used SRS devices. Of the nearly 135 data fields collected, only 25 fields require manual entry by a clinician. The lessening of the data-entry burden on clinicians will only help to increase the completeness, accuracy, and comprehensiveness of the national registry.

TABLE 1. Stereotactic radiosurgery (SRS)

\begin{tabular}{|c|c|c|c|c|c|}
\hline No. & Field Name & Data List & Data Type & & Comments \\
\hline 121 & System Treatment ID & & Guid & Single & Unique identifier of SRS Treatment \\
\hline 76 & Treatment Type & See "Treatment Types" & Guid & Single & \\
\hline 119 & System Patient ID & See "Patient" & Unsigned integer & Single & Unique identifier of Patient \\
\hline $\begin{array}{l}17 \\
18\end{array}$ & Diagnoses and Locations & $\begin{array}{l}\text { See "Patient Diagnosis" and } \\
\text { "Diagnosis Location" }\end{array}$ & $<$ Guid, Guid> & Multiple & \\
\hline 75 & $\begin{array}{l}\text { Date of Treatment (first Fraction) } \\
\quad(\text { MM-DD-YYYY) }\end{array}$ & & DateTime & Single & \\
\hline 114 & Neurosurgeon & See "Physician" & Guid & Single & \\
\hline 77 & Treatment Device Type & See "Treatment Device Type" & Guid & Single & \\
\hline 78 & Beam-Limiting Device & See "Beam Limiting Device Type" & Guid & Single & \\
\hline 80 & Cone Diameter (mm) & & Nullable Decimal (3.2) & Multiple & If Beam-Limiting Device is "Cones" \\
\hline 79 & MLC Leaf Size (mm) & & Nullable Decimal (3.2) & Multiple & If Beam-Limiting Device is "MLC" \\
\hline 81 & MLC Radiation Technique & See "Radiation Technique Type" & Nullable Guid & Single & If Beam-Limiting Device is "MLC" \\
\hline 81.1 & Other MLC Radiation Technique & & Nullable String (100) & Single & $\begin{array}{l}\text { If "MLC Radiation Technique" = } \\
\text { "Other" }\end{array}$ \\
\hline 82 & Immobilization System used & See "Immobilization System Type" & Guid & Single & \\
\hline 83 & Positioning Technology used & See "Position Technology Type" & Guid & Multiple & \\
\hline 83.1 & $\begin{array}{l}\text { Other Positioning Technology } \\
\text { used }\end{array}$ & & Nullable String (100) & Single & $\begin{array}{l}\text { If "Positioning Technology used" = } \\
\text { "Other" }\end{array}$ \\
\hline 84 & Position Reference & See "Position Reference Type" & Guid & Single & \\
\hline 84.1 & Other "Position Reference" & & Nullable String (100) & Single & If "Position Reference" = "Other" \\
\hline 85 & Position Monitoring & See "Position Monitoring Type" & Guid & Single & \\
\hline 86 & Treatment Planning System & $\begin{array}{l}\text { See "Treatment Planning System } \\
\text { Type" }\end{array}$ & Guid & Single & \\
\hline 86.1 & Other Treatment Planning System & & Nullable String (100) & Single & $\begin{array}{l}\text { If "Treatment Planning System" = } \\
\text { "Other" }\end{array}$ \\
\hline 87 & Total beam-on time (min) & & Decimal (4.2) & Single & \\
\hline 88 & Number of fractions & & Unsigned integer & Single & \\
\hline 89 & Steroids (adjuvant) & & Boolean & Single & \\
\hline 90 & Antiepileptics (adjuvant) & & Boolean & Single & \\
\hline 91 & Months until follow-up & & Unsigned integer & Single & \\
\hline \multirow[t]{3}{*}{134} & Comments & & Nullable String (300) & Single & \\
\hline & CTVs & See "CTV DESCRIPTION" & Guid & Multiple & \\
\hline & OARs & See "OAR DESCRIPTION" & Guid & Multiple & \\
\hline
\end{tabular}


At the time of entry, data elements will be checked to ensure that they are within preset reasonable ranges for each field. For example, a dose of 500 Gy will prompt a system response to query the appropriateness of the entry given that it falls outside the range of normal radiosurgical doses. In addition, auditing of the data by an independent clinical trials organization, Quintiles, was put into place to ensure the fidelity of the data. Also, Quintiles will perform queries to sites regarding any missing or seemingly erroneous data, which is important for increasing confidence of outside stakeholders in the reliability of data reported from this registry.

Also, a comprehensive set of rules for the governance of the quality and outcome data gathered as part of the registry was established. Outcomes will need to be risk adjusted rather than simply compared according to mean performance. Clinicians can access their data and compare them with an aggregate of their peers for self-assessment. However, more global access to the data is permitted through a process that requires a scientific proposal and review of the same by the SRS board. Quality and clinical research projects can be undertaken through a process that provides fairness and opportunity for all investigators while ensuring scientific oversight and biostatistical assistance to the endeavors. A firewall exists between corporate partners and the data repository.

The development of the previously successful lumbar spine registry was based upon a subscription model. Thus, clinician users or the health systems for which they worked paid for use of the online database. They also paid for the staff required to enter data into the registry. Whereas such a model proved successful, at least in the short term, for the lumbar spine registry, the model did not seem ideal for a national SRS registry. Moreover, lumbar spine registry fields were populated through manual data entry, which is laborious and prone to error.

Working with corporate partners including Brainlab and later Elekta, a funding model for the registry was established through corporate educational grants to the parent organizations. Other corporate partners have also expressed interest in the registry effort. This level of funding for the SRS registry eliminates the need for a subscription charge to enter data into the registry. The registry also provides funding to pilot sites to offset direct expenses for data collection, much like a funded clinical trial would do. Such a financial strategy represents a novel funding mechanism and unique set of partnerships that may represent a model to inform the design of other observational studies. It should help to ensure early and more widespread adoption of the registry and improved data fidelity. Also, with their extensive software-engineering experience, the corporate partners created data entry and extraction platforms that were compatible with the majority of SRS devices. Such platforms incorporated features including semiautomated data extraction from the SRS planning software and sophisticated neuroimaging analytics for follow-up assessment of SRS responses (Fig. 1). An organizational diagram of the SRS registry is depicted in Fig. 2.

At the onset, the registry will include 30 high-volume SRS sites (Table 2). Each of these sites has a volume of more than 100 intracranial SRS cases annually. The sites were selected in part to ensure geographic diversity of patient accrual and to reflect academic, private, and hybrid sites (Fig. 3). Also, a clinical investigator at each site had to express willingness to participate in the registry. The sites will accrue patients in Year 1 and test the electronic data-capturing software in a fashion that will no doubt lead to further refinements. In Year 2 of the effort and beyond, the SRS registry effort will be expanded further to open additional sites for accrual.

It is anticipated that by Year 3, data from 27,000 patients will have been accrued to the SRS registry, which would make this registry the largest and most comprehensive extant database for SRS clinical outcomes. The

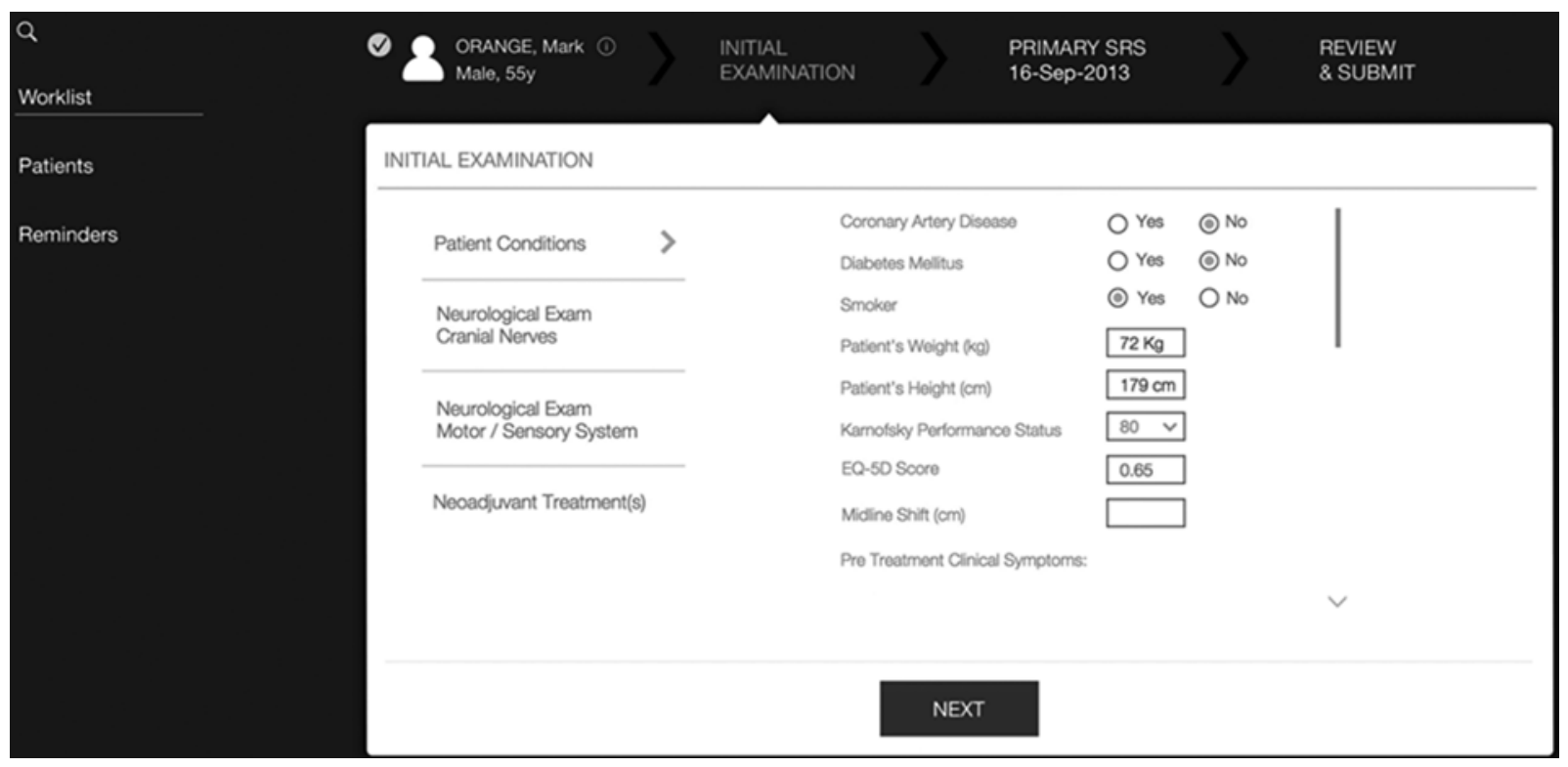

FIG. 1. Depiction of an SRS registry platform with representative data fields. The ease of manual data entry and the population of certain fields in a semiautomated or automated fashion should facilitate completeness and validity of the registry. 


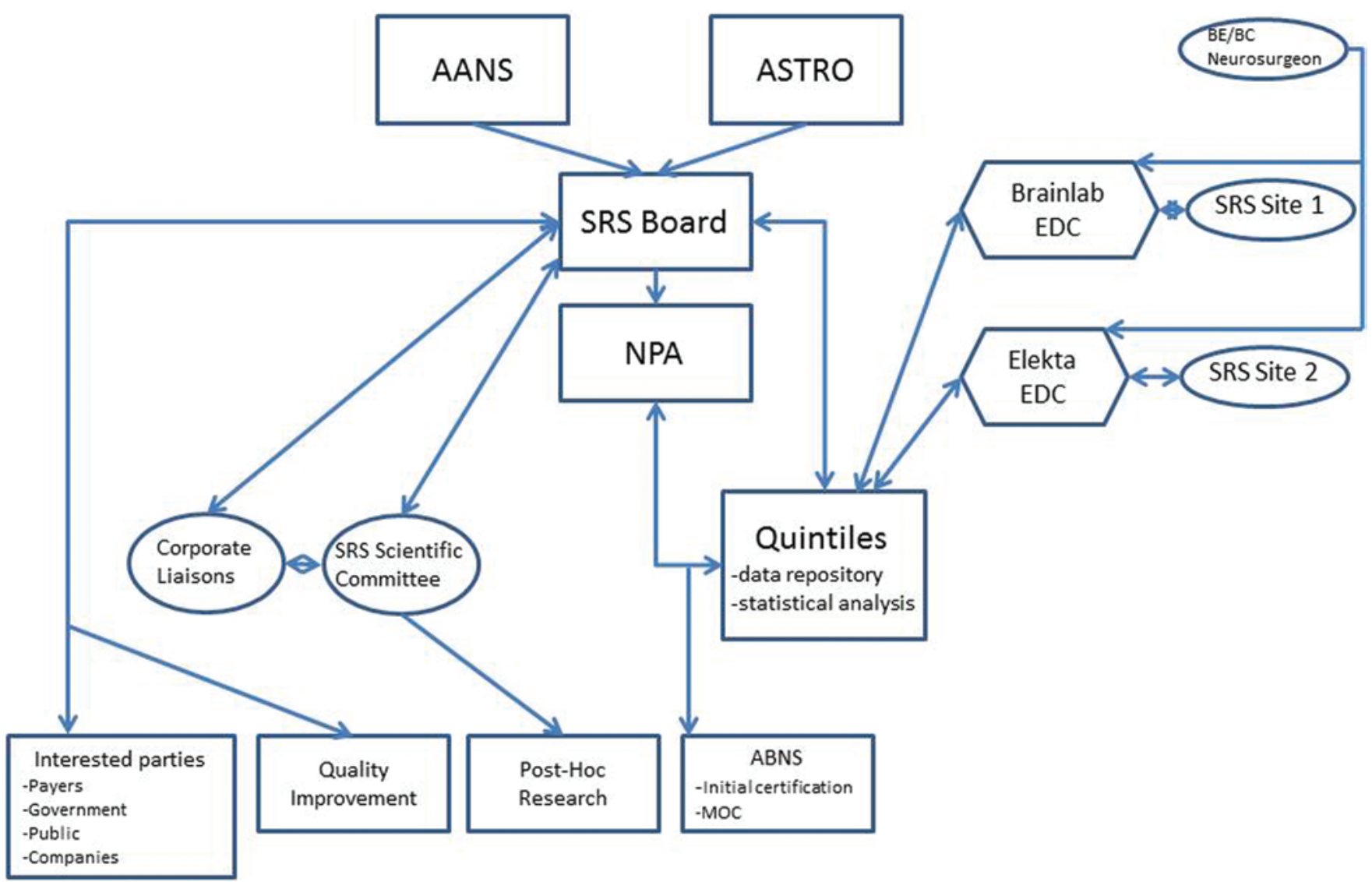

FIG. 2. Organizational diagram of the national SRS registry. BE/BC = Board eligible/Board certified; $E D C=$ electronic data collection. Figure is available in color online only.

statistical power of such a registry is appreciable. Of these 27,000 patients, if we assume that 5000 patients have an indication such as brain metastases and that $50 \%$ of patients undergo a particular SRS intervention with a more common outcome event (e.g., 25 per 100 patients), then according to a log-rank test, the study would have $90 \%$ power to detect a hazard ratio of 1.22 , assuming a 2 -sided test and alpha value of 0.05 . For a rarer outcome event (e.g., 1 per 100 patients), the same log-rank test would indicate a $90 \%$ power to detect a hazard ratio of 4.02 with 5000 patients, again assuming a 2 -sided test and an alpha value of 0.05 . Thus, the registry should offer "big-data" analytical options for clinicians who perform SRS.

At the end of Year 3 of the registry effort, the aggregate data will be in the public domain and provide health care consumers and other interested parties the opportunity to review and assess the outcomes associated with contemporary SRS.

\section{Maintenance of Certification and the SRS Registry}

The American Board of Medical Specialties is composed of representatives from individual medical boards including the American Board of Neurological Surgery (ABNS). In 2000, the American Board of Medical Specialties introduced continuous professional learning and development in an initiative called maintenance of certifi- cation (MOC). The ABNS MOC process includes evaluation of clinical outcomes derived from the self-reported details of 10 neurosurgical cases spanning a set period. ${ }^{1}$ The ABNS is presently involved in a reorganization of neurosurgical MOC, particularly MOC Part IV (practice performance). The new MOC process will involve incorporation of multiple novel methods to continuously advance quality improvement, including using patient care registries. The NPA serves as the data-collection partner for the ABNS. Patient-centered and risk-adjusted clinical outcomes can provide the ABNS the ability to assess a neurosurgeon's clinical skills, safety, and quality in his or her practice setting. The integration of the SRS registry into MOC would bring a level of objectivity and subspecialty insight to the assessment of an SRS practice, adding to the value of the MOC process in neurosurgery.

The SRS registry will comprise "real-world" data pertaining to outcomes. Comparing clinicians' outcomes and benchmarking individual surgeons' risk-adjusted outcomes may be an important addition to the ABNS MOC process. The key cases in radiosurgery can be benchmarked more accurately against the larger, aggregate outcomes derived from the national SRS registry. In particular, stratification of outcomes based on key prognostic factors (e.g., intracranial tumor volume, location, and neurological performance status) can provide better comparative assessments of performance. In this fashion, 
TABLE 2. The 30 pilot sites for the National SRS Registry

\begin{tabular}{|c|c|c|c|}
\hline No. & SRS Registry Site & City & State \\
\hline 1 & Barrow Neurological Institute, St. Joseph's Hospital and Medical Center & Phoenix & $A Z$ \\
\hline 2 & Palo Alto Medical Foundation & Palo Alto & $\mathrm{CA}$ \\
\hline 3 & Ronald Reagan UCLA Medical Center & Los Angeles & $\mathrm{CA}$ \\
\hline 4 & UCSF Medical Center & San Francisco & $\mathrm{CA}$ \\
\hline 5 & University of Southern California & Los Angeles & $\mathrm{CA}$ \\
\hline 6 & University of Colorado Health System, Poudre Valley Hospital & Fort Collins & $\mathrm{CO}$ \\
\hline 7 & Yale New Haven Health System & New Haven & CT \\
\hline 8 & UF Health Cancer Center at Orlando Health & Orlando & $\mathrm{FL}$ \\
\hline 9 & IU Health Neuroscience Center & Indianapolis & IN \\
\hline 10 & Norton Cancer Institute & Louisville & KY \\
\hline 11 & Vanderbilt & Nashville & TN \\
\hline 12 & William Beaumont Hospital & Royal Oak & $\mathrm{Ml}$ \\
\hline 13 & Carolinas Medical Center & Charlotte & NC \\
\hline 14 & Duke Cancer Center & Durham & NC \\
\hline 15 & Memorial Sloan Kettering Cancer Center & New York & NY \\
\hline 16 & NYU Langone Medical Center & New York & NY \\
\hline 17 & North Shore-LIJ Cancer Institute & New York & NY \\
\hline 18 & University of Rochester Medical Center, Strong Memorial Hospital & Rochester & NY \\
\hline 19 & NewYork-Presbyterian/Weill Cornell Medical Center & New York & NY \\
\hline 20 & University of Cincinnati; Mayfield Clinic & Cincinnati & $\mathrm{OH}$ \\
\hline 21 & Case Western Reserve University & Cleveland & $\mathrm{OH}$ \\
\hline 22 & Jefferson Hospital for Neuroscience & Philadelphia & PA \\
\hline 23 & Penn State Hershey Medical Center & Hershey & PA \\
\hline 24 & University of Pittsburgh Medical Center (Presbyterian/Shadyside) & Pittsburgh & PA \\
\hline 25 & Semmes-Murphey & Memphis & TN \\
\hline 26 & Moffitt Cancer Center & Tampa & $\mathrm{FL}$ \\
\hline 27 & Northwestern University & Evanston & IL \\
\hline 28 & UT Southwestern Medical Center & Dallas & TX \\
\hline 29 & Huntsman Cancer Institute University of Utah & Salt Lake City & UT \\
\hline 30 & University of Virginia Health System & Charlottesville & VA \\
\hline
\end{tabular}

the ABNS MOC process has the potential to lead to quality improvements by clinicians.

\section{Future Directions}

Radiosurgery represents a paradigm-shifting approach for the disciplines of neurosurgery and radiation oncology. The field continues to evolve in terms of the technology used to deliver it, and the indications for which it is used continue to expand. Over the coming years, the national SRS registry will evolve to meet the needs of a changing field and better serve the clinicians and patients whom it encompasses.

There are some diagnostic gaps in the current SRS registry. For less common indications such as essential tremor, cavernous malformations, gelastic seizures associated with hypothalamic hamartomas, and obsessive compulsive disorder, radiosurgery has shown considerable benefit for selected patients. However, the registry was not initially designed to capture data from patients with these types of diagnoses. The failure to include these rare indi- cations was not inadvertent but intentional. The volumes of patients with these SRS indications are usually low, and the resources required to track their outcomes are often distinctly different from those for other more standard SRS indications. However, these SRS indications remain important ones. Given their rarity, some of these SRS indications may ironically benefit the most from a big-data initiative such as this registry. Quality and outcome improvements can be challenging to achieve when centers encounter such cases only a few times per year. However, by pooling the data, benchmarking for outcomes can help to identify the benefit-to-risk profile for a procedure. The identification of prognostic factors for rare SRS indications can also lead to improved patient selection and appropriate allocation of medical resources. Outcome data from the registry can also lead to more rapid recognition of the value of an SRS procedure and the widespread adoption of the procedure for a newer indication.

Stereotactic radiosurgery is also a field that extends well beyond the border of the United States. In fact, radiosurgery is practiced quite frequently in developed countries 


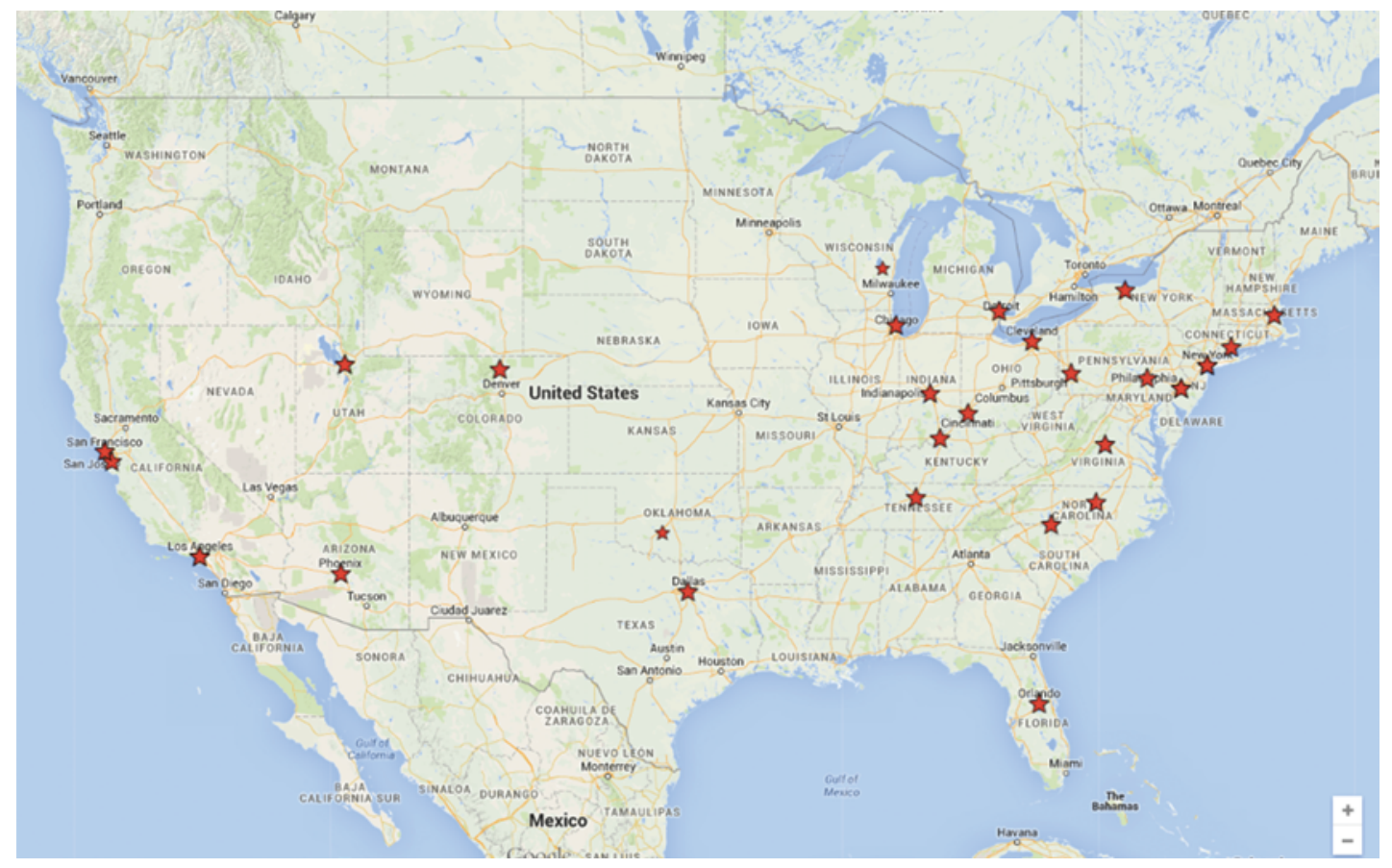

FIG. 3. Map depicting the geographic distribution of the SRS registry sites. The stars represent locations of the 30 pilot sites. Figure is available in color online only.

such as Japan and France. It is also permeating countries such as those in Africa. ${ }^{10}$ The platform, including the data dictionary, the data-entry system, analytics, and the secure repository, is Internet based. The cost of building registries has already largely been paid for through corporate grants and direct support from the 2 professional societies. Expanding the registry's reach beyond the United States to international partners may require a modest investment to scale it up. Accrual of international data would provide even "bigger" data for quality, outcome, and post hoc research. Opportunities for data-driven practice improvement using real-world clinical outcomes could benefit US and international SRS practitioners. Innovations in radiosurgery may be recognized more quickly with the statistical power of a large cohort, and problems associated with a particular technique could also be identified more easily and revised accordingly. Ongoing discussions with international clinical partners about expanding the SRS registry are underway.

Finally, the SRS registry requires a financial model that will enable it to exist in perpetuity. Such a model will continue to require modest investments from corporate partners, clinicians, and professional societies. However, the sources of support for the registry can be diversified to include other groups such as private payers, patients, foundations, and the federal government. The cost of continuing the final registry pales in comparison with the comparable cost of a single RCT.

\section{Conclusions}

The future of registries in US clinical medicine seems to be a bright one. The multidisciplinary nature of the SRS registry and the financial model that enables its implemen- tation may serve as models for future registry efforts. The real-world data accrued through the registry will help to advance the field of SRS. Quality improvement, innovation, and cost-effective care should be enhanced to the benefit of patients and the clinicians treating them.

\section{Acknowledgment}

We appreciate the statistical guidance by Dr. William Hawkes of Quintiles.

\section{References}

1. American Board of Neurological Surgery: Maintenance of Certification Handbook. Woodbridge, CT: American Board of Neurological Surgery, 2013

2. Asher AL, McCormick PC, Selden NR, Ghogawala Z, McGirt MJ: The National Neurosurgery Quality and Outcomes Database and NeuroPoint Alliance: rationale, development, and implementation. Neurosurg Focus 34(1):E2, 2013

3. Barnett GH, Linskey ME, Adler JR, Cozzens JW, Friedman WA, Heilbrun MP, et al: Stereotactic radiosurgery-an organized neurosurgery-sanctioned definition. J Neurosurg 106:1-5, 2007

4. Betti OO: [History of radiosurgery.] Cancer Radiother 2:101-104, 1998 (Fr)

5. Betti O, Derechinsky V: [Multiple-beam stereotaxic irradiation.] Neurochirurgie 29:295-298, 1983 (Fr)

6. Colombo F, Benedetti A, Pozza F, Avanzo RC, Marchetti C, Chierego G, et al: External stereotactic irradiation by linear accelerator. Neurosurgery 16:154-160, 1985

7. Concato J: Is it time for medicine-based evidence? JAMA 307:1641-1643, 2012

8. Dahlin H, Larsson B, Leksell L, Rosander K, Sarby B, Steiner L: Influence of absorbed dose and field size on the geometry of the radiation-surgical brain lesion. Acta Radiol Ther Phys Biol 14:139-144, 1975 
9. Efstathiou JA, Nassif DS, McNutt TR, Bogardus CB, Bosch W, Carlin J, et al: Practice-based evidence to evidence-based practice: building the National Radiation Oncology Registry. J Oncol Pract 9:e90-e95, 2013

10. Fezeu F, Awad AJ, Przybylowski CJ, Starke RM, Grober Y, Schlesinger D, et al: Access to stereotactic radiosurgery: identification of existing disparities and a modest proposal to reduce them. Cureus 6:e157, 2014

11. Hartmann GH, Schlegel W, Sturm V, Kober B, Pastyr O, Lorenz WJ: Cerebral radiation surgery using moving field irradiation at a linear accelerator facility. Int J Radiat Oncol Biol Phys 11:1185-1192, 1985

12. Leksell L: The stereotaxic method and radiosurgery of the brain. Acta Chir Scand 102:316-319, 1951

13. Lutz W, Winston KR, Maleki N: A system for stereotactic radiosurgery with a linear accelerator. Int J Radiat Oncol Biol Phys 14:373-381, 1988

14. McGirt MJ, Speroff T, Dittus RS, Harrell FE Jr, Asher AL: The National Neurosurgery Quality and Outcomes Database (N2QOD): general overview and pilot-year project description. Neurosurg Focus 34(1):E6, 2013

15. Podgorsak EB, Olivier A, Pla M, Hazel J, de Lotbinière A, Pike B: Physical aspects of dynamic stereotactic radiosurgery. Appl Neurophysiol 50:263-268, 1987
16. Seung SK, Larson DA, Galvin JM, Mehta MP, Potters L, Schultz CJ, et al: American College of Radiology (ACR) and American Society for Radiation Oncology (ASTRO) Practice Guideline for the Performance of Stereotactic Radiosurgery (SRS). Am J Clin Oncol 36:310-315, 2013

17. Stewart DJ, Kurzrock R: Fool's gold, lost treasures, and the randomized clinical trial. BMC Cancer 13:193, 2013

\section{Disclosure}

The authors report no conflict of interest concerning the materials or methods used in this study or the findings specified in this paper.

\section{Author Contributions}

Conception and design: Sheehan. Drafting the article: Sheehan. Critically revising the article: all authors.

\section{Correspondence}

Jason P. Sheehan, Department of Neurological Surgery, University of Virginia, Box 800212, Charlottesville, VA 22908. email: jsheehan@virginia.edu. 